Société d'histoire de la révolution de 1848 et des

révolutions du XIXe siècle

$47 \mid 2013$

Quel est l'avenir du XIX $X^{\mathrm{e}}$ siècle ?

\title{
Un passé qui ne passe pas
}

Isabelle Garo

URL : http://journals.openedition.org/rh19/4533

DOI : $10.4000 /$ rh19.4533

ISSN : 1777-5329

Éditeur

La Société de 1848

Édition imprimée

Date de publication : 31 décembre 2013

Pagination : 15-19

ISSN : 1265-1354

Référence électronique

Isabelle Garo, «Un passé qui ne passe pas », Revue d'histoire du XIXe siècle [En ligne], 47 | 2013, mis en ligne le 31 décembre 2016, consulté le 30 avril 2019. URL : http://journals.openedition.org/rh19/4533 ; DOI : 10.4000/rh19.4533 
ISABELLE GARO

\section{Un passé qui ne passe pas}

Travailler sur Marx, c'est forcément s'intéresser au XIX ${ }^{e}$ siècle, à ses séquences révolutionnaires, mais aussi à l'expansion du capitalisme, ces deux fils se tressant l'un à l'autre jusqu'à aujourd'hui. Être marxiste pose un tout autre problème : comment est-il concevable qu'un penseur du XIX ${ }^{e}$ siècle puisse nous parler encore? La question n'est pas neuve. Mais il faut en ajouter une seconde, qui la complique et la précise : l'actualité du XIX ${ }^{\mathrm{e}}$ siècle se trouve assurément du côté des outils d'analyse du capitalisme, qui sont forgés alors même que ce mode de production entre dans sa phase industrielle mature. Mais elle se trouve aussi du côté de la volonté politique montante de son abolition et de la naissance des organisations qui inscrivent le socialisme et le communisme sur leurs bannières.

En ce sens, il faut reconnaître que le XIX ${ }^{\mathrm{e}}$ siècle concerne, comme aucun autre siècle sans doute, à la fois notre passé, notre présent et notre avenir. Par les crises qui le taraudent depuis cette époque, le capitalisme se transforme en permanence tout en se bouclant sur sa propre logique contradictoire, inventant une histoire qui mêle reproduction et ruptures, innovations et répétitions. Le rapport que l'on peut entretenir avec le XIX ${ }^{\mathrm{e}}$ siècle, depuis le XXI ${ }^{e}$ commençant lui-même par une crise majeure, pour ne pas être le rabattement illusoire d'un temps sur un autre, doit prendre la figure de ce repli décalé, dialectique, de l'histoire réelle sur ses contradictions toujours singulières, qui stratifie à la fois le cours des choses, ses lectures historiques autant que les projections transformatrices. Que l'historiographie autant que l'histoire du $\mathrm{XIX}^{\mathrm{e}}$ soient un champ de bataille est donc la moindre des choses.

Pour le dire schématiquement, deux façons d'envisager le XIX ${ }^{\mathrm{e}}$ siècle depuis le XXI e sont possibles. Face aux tentatives, aux désastres et aux échecs du XX $X^{e}$ siècle concernant le dépassement du capitalisme, alors qu'il est de mise d'en omettre les réussites et les conquêtes, on peut souhaiter revenir à une inspiration première. L'intérêt contemporain pour Marx mais aussi pour Proudhon, Fourier, Blanqui, Saint-Simon, pour la littérature ouvrière, pour 1848 et pour la Commune de Paris, prouve que les projets et les mobilisations politiques de ce siècle restent et reviennent au centre des préoccupations 
d'aujourd'hui. Pourtant, et c'est une deuxième option, on peut juger illusoire de vouloir revivifier une origine, elle-même en proie à ses contradictions datées, en ne prenant plus en compte que des textes et des programmes, omettant les échecs, oubliant un peu vite les conditions de vie et de travail d'alors.

C'est donc à travers le $\mathrm{XX}^{\mathrm{e}}$ siècle et à partir de ce qu'est le XXI ${ }^{\mathrm{e}}$ commençant qu'il est fécond de réfléchir aux idées et aux événements d'il y a cent-cinquante ans et plus. Il ne s'agit alors pas de revenir à des potentialités dépassées - tout possible s'enracinant dans son présent -, mais de s'interroger sur des processus qui se poursuivent et sur des filiations qui, de ce fait, se transforment. La question de l'actualité du XIX siècle, et de ce qui y est actualisable, est donc une question complexe, qui exige d'être dialectisée pour ne pas se réduire au pur rappel rhétorique d'un passé plein d'avenir : les contradictions réelles sont propres à chaque séquence historique, bien entendu, mais elles tiennent, de ce fait aussi, à la réception de cette histoire par la période qui la suit, en ses clivages politiques et sociaux, qui y puise, y projette ou y confronte la conscience de ses propres contradictions et ses propres aspirations. Les anarchistes ont leur XIX ${ }^{e}$ siècle, les libéraux en ont un autre.

À partir de là, et si l'on privilégie la dimension profondément politique de la question, deux points interpellent tout particulièrement ceux qui se revendiquent aujourd'hui de Marx et du marxisme. Le premier concerne le capitalisme en tant que mode de production qui relie le XIX ${ }^{e}$ au XXI ${ }^{e}$ siècle, par continuités et différences. Le second concerne les perspectives politiques et, de ce fait, la question communiste notoirement résurgente, mais principalement sur le terrain théorique pour le moment.

Le capitalisme du XIX ${ }^{\mathrm{e}}$ siècle n'est pas le nôtre, pour bien des raisons. Pourtant, il installe une logique économique et sociale d'ensemble qui reste celle du capitalisme d'aujourd'hui : transformation de l'agriculture, exploitation du travail et division poussée de celui-ci, salariat, propriété privée des grands moyens de production, mutations technologiques, perfectionnement des moyens de transport, accélération et mondialisation des échanges, formation des classes et fractions de classe modernes, luttes sociales et politiques, rapports mondiaux de domination, colonialisme, impérialisme, crises. L'industrialisation qui se produit en Europe et aux États-Unis inaugure et met en action des forces productives nouvelles, gigantesques, et des conditions de travail inédites, nées de la mutation de formes antérieures de production, initialement marquées par le féodalisme puis restructurées en profondeur.

Selon les lectures adoptées, toutes politiques, la révolution industrielle est la conséquence de l'expansion de longue durée du marché, ou bien elle est le résultat d'un ensemble d'innovations technologiques, ou bien encore elle résulte d'une transformation d'ensemble du mode de production qui débute par la modification des rapports sociaux au sein de l'agriculture anglaise. 
Selon les approches, on analysera autrement les transformations des forces productives contemporaines : la montée des technologies de l'information et de la communication sont-elles le point de départ d'un renouvellement du régime d'accumulation du capital? Pour les tenants du capitalisme cognitif, de nouveaux rapports sociaux, égalitaires, sont en germe dans les conditions contemporaines du travail immatériel. Pour d'autres, les rapports d'exploitation demeurent et se renouvellent à travers des technologies qui ne sont pas par elles-mêmes porteuses de renouveau social ou politique. La question n'est pas neuve et la force prédictive des thèses marxiennes se trouve aussi là, où on l'attend le moins.

On peut en dire autant des crises économiques : le XIX siècle voit naître les premières grandes crises périodiques du capitalisme. La plus grave, au cours du siècle suivant, celle qui débute en 1929, aura pour conséquence, en particulier après la Seconde Guerre mondiale qui en est inséparable, la mise en place d'un nouveau régime d'accumulation et de politiques sociales étatiques, redistributives jusqu’à un certain point. Cette période, qui se clôt sous nos yeux, ne fut qu'une parenthèse : la présente crise, accompagnée des politiques néolibérales qui l'approfondissent et qui restaurent des rapports de classes d'un autre âge, ne renvoie sans doute pas au XIX siècle. D'abord parce que l'histoire ne revient pas sur ses pas. Ensuite, en raison de la gravité de la crise actuelle, qui retourne les promesses en menaces : le capitalisme d'aujourd'hui, qui a conquis la planète, ne peut plus opter, désormais, que pour le renforcement généralisé de l'exploitation, la mise en concurrence des travailleurs, la casse de l'État social quand il existe, les tensions internationales et la militarisation du monde, et une financiarisation de l'économie sans précédent, indissociable du régime actuel d'accumulation et de ses tâtonnements en quête de stabilité. En revanche, le durcissement de l'affrontement de classe ainsi que l'affaiblissement du mouvement ouvrier, les dérégulations en cours, nous rapprochent à l'évidence de ce que fut le moment de l'installation des rapports capitalistes, et de sa violence. Une logique nue, celle du profit et de la loi de la valeur, se conçoivent mieux aujourd'hui en lisant le Capital plutôt que les œuvres des keynésiens ou des régulationistes.

L'erreur, à partir de là, ce serait de prendre les parentés pour une identité : c'est une généalogie qu'il importe de reconstituer, des causes historiques qu'il faut identifier et cet effort renvoie une fois de plus aux questions les plus contemporaines. Il faut les considérer sous l'angle politique. D'abord parce que lui seul autorise un rapprochement qui se sait et s'avoue construit, et qu'éclaire la volonté de transformation du présent, loin que le passé nous prescrive ou nous conseille quoi que ce soit. Ensuite, parce que l'actualité du $\mathrm{XIX}^{\mathrm{e}}$ tient à ce siècle des révolutions qu'il fut, en relation jamais mécanique mais en relation toutefois avec les conditions capitalistes de la production, avec les crises économiques et sociales qui en résultèrent, et dont les convulsions présentes sont une réplique bien plus forte que les séismes initiaux. 
Pourtant, aucun modèle abstrait n'en découle, et surtout pas la perspective d'un effondrement du capitalisme. La pertinence du rapprochement tient alors surtout à l'urgence de l'intervention politique, selon ses paramètres propres, sans recette ni garantie.

En effet, les révolutions qui ponctuent le $\mathrm{XIX}^{\mathrm{e}}$ siècle, aussi différentes soient-elles les unes des autres, voient se structurer les luttes sociales et se former les organisations politiques et syndicales modernes. Il est bien évident que nous sommes les héritiers de ces formes mais aussi les héritiers de leur déclin relatif, et à coup sûr de leur crise profonde. Or, c'est bien le retour du capitalisme à son régime de crise, par delà les tentatives provisoires et finalement manquées de sa régulation étatique, qui nous renvoie cette question cruciale : comment construire des organisations, porteuses du projet d'une tout autre organisation économique et sociale, qui soient des forces syndicales et politiques échappant à l'autoritarisme, à la bureaucratie et aux hiérarchies traditionnelles, et qui portent enfin victorieusement le projet d'une sortie décidée hors du capitalisme? Paradoxe de plus, et non des moindres: l'histoire $\mathrm{du} \mathrm{XIX}^{\mathrm{e}}$ siècle oblige à nuancer considérablement le réquisitoire sans nuance souvent porté sur le $\mathrm{XX}^{\mathrm{e}}$ siècle finissant à ce sujet. Elle invite même à relire les épisodes du siècle passé, en rediscutant les condamnations qu'il énonça sur lui-même.

Finalement, et contre toute attente, l'importance du XIX ${ }^{e}$ siècle aujourd'hui tient aussi à ce rapport complexifié qu'il permet avec le $\mathrm{XX}^{\mathrm{e}}$ siècle. Bien des types d'organisation virent alors le jour, des plus libertaires aux plus autoritaires, des sectes blanquistes aux partis de masse, à l'exemple de la sociale-démocratie nord-européenne, des structures les plus locales à la Première Internationale, des luttes sociales aux luttes féministes ou indépendantistes. Et bien des révoltes, toutes défaites finalement, mais puissamment inventives, des Canuts à la Commune de Paris. Il ne s'agit pas tant de s'y ressourcer que de les connaître, à la fois pour ne pas bégayer, et pour en mesurer les acquis et les échecs. Et le mot de communisme, le plus typique qui soit du XIX ${ }^{\mathrm{e}}$ siècle, porte mieux que tout autre ces contradictions et ces projets renaissants, à condition de ne rien omettre de la diversité de ses emplois. La prise en compte de son épaisseur historique, sans simplification aucune, conditionne son actualité.

Car paradoxalement, dialectiquement surtout, l'actualité du communisme ne saurait provenir de son caractère supposé éternel, surplombant l'histoire, mais bien de son insertion dans le réel, qui oblige à le redéfinir à la fois selon les coordonnées du présent et dans la relation à son passé. La fameuse définition qu'en avaient donnée Marx et Engels en 1846, dans L'idéologie allemande, reste plus que jamais à prendre au sérieux : "Pour nous, le communisme n'est pas un état de choses qu'il convient d'établir, un idéal auquel la réalité devra se conformer. Nous appelons communisme le mouvement réel qui abolit l'état actuel des choses. Les conditions de ce mouvement 
résultent des données préalables telles qu'elles existent actuellement». Ces phrases, tout droit venues du XIX ${ }^{e}$ siècle, affirment par avance comme un paradoxe, et surtout comme une tâche, leur propre actualité : le communisme, s'il existe, est la nécessité de l'invention historique, sur la base de "conditions réelles», héritées du passé. Le XIX ${ }^{\mathrm{e}}$ siècle fait bien évidemment partie de ces "conditions réelles». Et sa connaissance disputée également.

Isabelle Garo est philosophe; elle préside la Grande Édition des Euvres de Marx et d'Engels en français (GEME-Les Éditions sociales). Elle a notamment publié Marx, une critique de la philosophie, Le Seuil, 2000 et Marx et l'invention historique, Syllepse, 2012 